\title{
Performances agronomiques et stabilité phénotypique de génotypes de Sorgho (Sorghum bicolor (L.) Moench) au Sénégal : une étude des interactions génotypes-environnement
}

\author{
Malick NDIAYE ${ }^{1,2^{*}}$, Myriam ADAM ${ }^{3,6,7}$, Bertrand MULLER $2,3,4$, Aliou GUISSE ${ }^{5}$, Ndiaga CISSE2 \\ 1. Institut Sénégalais de Recherches Agricoles (ISRA), CRA de Saint-Louis, Route de Leybar B.P. 240 \\ Saint-Louis, Sénégal \\ 2. ISRA/Centre d'Étude Régional pour l'Amélioration de l'Adaptation à la Sécheresse (CERAAS), B.P. 3320 Thiès- \\ Escale, Sénégal \\ 3. UMR-AGAP, Univ Montpellier, CIRAD, INRA, Montpellier SupAgro, B.P. 34398 Montpellier, France \\ 4. CIRAD, UMR AGAP, 101 Antananarivo, Madagascar \\ 5. Université Cheikh Anta Diop de Dakar, Avenue Cheikh Anta Diop, BP 5005 Dakar, Sénégal \\ 6 . International Crops Research Institute for the Semi-arid Tropics (ICRISAT), BP320, Bamako, Mali \\ 7. Institut National de l'Environnement et de Recherches Agricoles (INERA), Bobo- Dioulasso, Burkina Faso. \\ Auteur correspondant : (*) agromalick@yahoo.fr
}

Original submitted in on $15^{\text {th }}$ February 2018. Published online at www.m.elewa.org on $31^{\text {st }}$ May 2018 https://dx.doi.org/10.4314/jab.v125i1.10

\section{RESUME}

Objectif : L'introduction de nouveaux génotypes de sorghos adaptés à divers environnements est confrontée à la présence d'interaction génotype $x$ environnements significative qui en réduise l'efficacité. La présente étude, conduite sur six environnements - combinaisons site-date de semis-année -, analyse l'interaction génotypeenvironnement et l'adaptabilité et stabilité du rendement grain et biomasse paille de 10 génotypes de sorgho (Sorghum bicolor (L.) Moench).

Méthodologie et résultats : L'essai a été conduit selon un dispositif en Blocs Complets Randomisés avec quatre répétitions. L'Anova combinée pour les rendements grains et biomasse paille a révélé une valeur hautement significative $(P<0,01)$ pour les génotypes, les environnements et leurs interactions. L'interaction significative a montré que les génotypes réagissent différemment dans les différents environnements. Les rendements moyens des génotypes ont varié de $1854 \mathrm{Kg}_{\text {ha- }}{ }^{-1}$ (Nieleni) à $547 \mathrm{Kg} \cdot \mathrm{ha}^{-1}(\mathrm{SK} 5912)$ pour le grain ; et de $12103 \mathrm{Kg}$ ha-1 $^{-1}$ (IS15401) à $4647 \mathrm{Kg} \mathrm{ha}^{-1}$ (CSM63E) pour la biomasse paille. Pour les environnements, les rendements ont varié de $1714 \mathrm{Kg}_{\text {ha }}{ }^{-1}$ (S13D1) à $530 \mathrm{Kg}^{\prime} \mathrm{ha}^{-1}(\mathrm{~B} 13 \mathrm{D} 2)$ pour le grain ; et de $9642 \mathrm{Kg} \cdot \mathrm{ha}^{-1}$ (B13D2) à $5742 \mathrm{Kg} . h a-1^{-1}$ (S13D2) pour la biomasse paille. L'analyse AMMl a montré que de bons rendements grains été associés avec des environnements à bonnes fertilité de sol et une bonne pluviométrie, alors que les rendements biomasse paille été plus influencés par la date de semis et la pluviométrie. De même, nous avons pu confirmer pour nos 10 génotypes de sorghos que la stabilité des rendements est en général associée avec de faibles performances, à l'exception du génotype Nieleni qui a de bonnes performances en grain et biomasse paille indépendamment de l'environnement. 


\title{
Ndiaye et al, J. Appl. Biosci. 2018 Performances agronomiques et stabilité phénotypique de génotypes de
} Sorgho (Sorghum bicolor (L.) Moench) au Sénégal : une étude des interactions génotypes-environnement

Conclusion et applications des résultats : Les environnements ont provoqués des réponses différentes des génotypes et la plupart de ces génotypes ont montré une spécificité environnementale. Cette étude trouve son application en sélection variétale et en agronomie. Elle pourrait non seulement aider les programmes de sélection dans le choix des stratégies de sélection pour l'amélioration des rendements en exploitant positivement l'interaction sur les sites à haut potentiel de rendement (S13D1 et S13D2) et l'adaptation générale aux sites au potentiel relativement plus faible (B13D1 et B13D2) mais aussi d'élargir la zone de culture du sorgho au Sénégal qui se fait sur sol argileux. En effet, les dix génotypes étudiés offrent aux producteurs une large gamme de choix variétal en fonction des conditions de cultures (sol et gestion) surtout Nieleni pour assurer leur production en grain et paille dans nos systèmes de production mixtes agriculture-élevage, où la double production est une option privilégiée.

Mots clés : Sorgho, Rendement, interaction génotype-environnement, stabilité, AMMI.

\section{Agronomic performance and phenotypic stability of Sorghum genotypes (Sorghum bicolor (L.) Moench)} in Senegal : a study of genotype-environment interactions

\begin{abstract}
Objective : The introduction of new genotypes of sorghums adapted to different environments is confronted with the presence of significant genotype $x$ environments interaction which reduces their effectiveness. The present study, conducted in six environments - site-date of sowing-year combinations -, analyzes the genotypeenvironment interaction and the adaptability and stability of grain and straw biomass yields of 10 sorghum genotypes (Sorghum bicolor (L.) Moench).

Methodology and Results : The experiment was laid down in a Randomized Complete Block Design with four replications. The combined ANOVA for grain and biomass straw yields revealed highly significant $(P<0.01)$ for genotypes, environments and their interactions. The significant interaction showed the genotypes respond differently across the different environments. The mean yields values of genotypes ranged from $1854 \mathrm{Kg}^{-h^{-1}} \mathrm{f}^{-1}$ (Nieleni) to $547 \mathrm{Kg} \mathrm{ha}^{-1}$ (SK5912) for grain ; and from $12103 \mathrm{Kg} \mathrm{ha}^{-1}$ (IS15401) to $4647 \mathrm{Kg} \mathrm{ha}^{-1}$ (CSM63E) for


and from $9642 \mathrm{Kg} \mathrm{ha}^{-1}$ (B13D2) to $5742 \mathrm{Kg} \mathrm{ha}^{-1}$ (S13D2) for straw biomass. The AMMl analysis showed that good grain yields were associated with good soil fertility environments and good rainfall, while biomass straw yields were more influenced by sowing date and rainfall. Similarly, we were able to confirm for our 10 sorghum genotypes that yield stability is generally associated with poor performance, with the exception of the Nieleni genotype which has good grain and biomass straw performance regardless of the environment.

Conclusion and application : This study application is in the varietal selection and in agronomy. It could not only assist breeding programs in choosing breeding strategies for yield improvement by positively exploiting the interaction at sites with high yield potential (S13D1 and S13D2) and general adaptation to sites with relatively lower potential (B13D1 and B13D2) but also to expand the area of cultivation of sorghum in Senegal which is done on clay soil. Indeed, the ten genotypes studied offer farmers a wide range of varietal choices depending on the growing conditions (soil and management) especially Nieleni to ensure their production in grain and straw in our mixed farming-livestock production systems, where the double production is a preferred option. Keywords: Sorghum, yield, genotype-environment interaction, stability, AMMI.
\end{abstract}

\section{INTRODUCTION}

Le sorgho (Sorghum bicolor (L.) Moench) est une des principales céréales cultivée dans les régions tropicales arides et semi-arides (Djè et al., 2007). Considéré comme une plante de région chaude, le sorgho, de par sa plasticité, est susceptible de croître aussi bien dans les régions tempérées que tropicales. Avec une production mondiale d'environ 68,9 millions de tonnes en 2015, sur environ 49,9 millions d'hectares, le sorgho est classé en cinquième position de la production céréalière après 

Sorgho (Sorghum bicolor (L.) Moench) au Sénégal : une étude des interactions génotypes-environnement

le maïs, le blé, le riz, et l'orge (FAOSTAT, 2015) et est principalement utilisé pour l'alimentation animale dans la plupart des pays développés. Mais en Afrique et en Inde, il constitue un aliment de base pour des millions de personnes (Agrama et Tuinstra, 2003). Par ailleurs, la culture du sorgho est l'une des plus importantes pouvant être utilisées pour la production de bioéthanol (Reddy et al., 2008). Au Sénégal, le sorgho est, après le mil, la deuxième céréale pluviale cultivée avec une superficie totale estimée à plus de 200737 ha pour une production nationale de 188500 tonnes et un rendement moyen de $939 \mathrm{Kg} / \mathrm{ha}$ (FAOSTAT, 2015). II constitue une des bases de l'agriculture vivrière de la population (Ba et al., 2010). Cependant sa production est confrontée à plusieurs contraintes qui entrainent des baisses de rendement (ou « des faibles rendements "), à savoir les irrégularités de la distribution des pluies, accentuées par le changement climatique, la faible fertilité des sols et leur caractère sableux, et diverses maladies et ravageurs des récoltes (Seguin et Soussana, 2008). Au Sénégal, parmi les actions en faveur de la sécurité alimentaire figurent la création et/ou l'introduction de nouveaux génotypes de sorghos adaptés à divers environnements en termes de sol et de climat. Cependant, lorsque des génotypes sont évalués en vue de faire des recommandations, un problème commun se pose :à savoir celui de la variation parfois élevée de leur productivité d'une année à l'autre et d'un environnement à l'autre. Cette variabilité crée une difficulté à déterminer quels génotypes peuvent être recommandés, et cette question mérite d'être examinée avec attention. La réponse différente d'un génotype dans différents environnements est connue sous le nom d'interaction génotype-environnement $(G \times E)$. Les possibles complications pouvant résultées d'éventuelles interactions $G \times E$ peuvent être limitées par l'identification de génotypes aux performances stables car adaptés à des conditions de culture assez diversifiées (Cruz et Regazzi,

\section{MATERIEL ET METHODES}

Site expérimentaux: Les expérimentations ont été menées lors des saisons des pluies 2013, 2014 dans deux stations de recherche de l'Institut Sénégalais de Recherches Agricoles (ISRA), à savoir les stations de
1997). Plusieurs méthodes statistiques ont été développées pour caractériser l'effet de l'interaction $G \times E$ des génotypes sélectionnés et pour prédire les réponses phénotypiques aux changements environnementaux. Cependant, la plupart des méthodes de stabilité statistique ne sont pas en mesure de fournir un modèle de réponse varié précis et complet pour cette interaction (Holhs, 1995), ce du fait surtout que la réponse génotypique à la variation environnementale est multi-variée et que plusieurs indices de stabilité ont une réponse uni-variée (Crossa, 1990). De nombreuses méthodes ont été développées pour exposer les modèles d'interaction $G \times E$. Parmi ceux-ci, le modèle des effets additifs et l'interaction multiplicative (AMMI) est une méthode multi-variée robuste pour les essais multienvironnementaux (Romagosa et Fox, 1993). La méthode d'analyse des effets additifs et de l'interaction multiplicative (AMMI) intègre l'analyse de variance (ANOVA) et l'analyse en composantes principales (PCA) dans une approche unifiée qui peut être utilisée pour analyser des essais multienvironnements (Zobel et al., 1988 ; Crossa et al., 1990, Gauch et Zobel, 1996) : I'ANOVA va étudier les principaux effets des génotypes et des environnements et la PCA porte sur la partie non additive du modèle $(G \times E)$. L'AMMI fournit la somme des carrés des écarts d'interaction $(G \times E)$ avec un nombre minimal de degrés de liberté. De plus, l'AMMI quantifie simultanément la contribution de chaque génotype et environnement à la somme des carrés des écarts de l'interaction $(G \times E)$, et fournit une interprétation graphique simple des résultats par la technique du graphe biplot pour classer simultanément les génotypes et les environnements (Kempton, 1984, Zobel et al., 1988). Notre étude présentée ici vise à analyser l'interaction génotypeenvironnement et l'adaptabilité et stabilité phénotypique de dix génotypes de sorghos au Sénégal, en utilisant la méthode AMMI.

Sinthiou Malem (en 2013, 2014) et de Bambey (en 2013). Les caractéristiques des différents sites expérimentaux sont données dans le tableau 1 et la figure 1 présente les évolutions des pluviométries et des températures durant 
les essais. La station de Bambey est soumise à un climat typiquement sahélien marqué par une longue saison sèche de 8 à 9 mois et une saison des pluies de 3 à 4 mois. Les sols dominants sont des sols sableux ayant une très faible capacité de rétention en eau de 90 à 100 mm. $\mathrm{m}^{-1}$ (Vachaud et al., 1978; Imbernon, 1981). La station Sinthiou Malem se situe à l'interface entre les zones sahéliennes et soudaniennes. Elle bénéficie d'une saison des pluies de 4 à 5 mois plus abondante qu'à Bambey, mais marquée par une variabilité interannuelle forte. Ses sols sont aussi à dominance sableuse mais présentent des taux d'argile et de limons légèrement plus élevés, inférant des capacités de rétention plus élevés.

Matériel végétal : Le matériel végétal est constitué de dix génotypes issus de régions diverses d'Afrique de l'Ouest et Centrale qui sont reconnus pour être chacun performant dans sa zone de diffusion. lls ont été retenus pour constituer un échantillon contrasté en ce qui concerne leurs durées de cycle (chacune étant adaptée à sa région cible), architectures (hauteur, diamètre de tige notamment), caractéristiques structurales (lignine, cellulose), et productions de grains et de biomasse. Les caractéristiques de ces dix génotypes sont présentées au tableau 2.
Conduite des essais : Pour chacun des sites, le travail de sol a consisté en un labour croisé aux disques (profondeur environ $25 \mathrm{~cm}$ ) suivi d'un hersage. Les semences ont été traitées au fongicide-insecticide Granox (association de captafol-bénomyl et de carbofuran). Les semis ont toujours été effectués après une bonne pluie (dates de semis au tableau 1). Les cultures ont été semées en poquets de 5-6 graines avec des écartements de $0,80 \mathrm{~m}$ entre ligne et $0,20 \mathrm{~m}$ sur la ligne, soit à une densité de 62500 poquets.ha ${ }^{-1}$. Le démariage s'est effectué à un plant, en condition humide, avant le tallage (environ 15ième jour). Une fertilisation minérale a été appliquée selon les recommandations de la recherche agronomique au Sénégal : épandage d'engrais N-P-K $(15 \% \mathrm{~N}, 15 \% \mathrm{P}, 15 \% \mathrm{~K})$ à la dose de $150 \mathrm{Kg}^{\mathrm{h}} \mathrm{ha}^{-1}$ au semis ou à la levée, et deux apports d'urée $(46 \% \mathrm{~N})$ à la dose de $50 \mathrm{Kg}$ ha-1, juste après le démariage pour le premier puis à la montaison pour le second. Des sarclobinages, des traitements phytosanitaires (Décis et Diméthoate) et une protection par gardiennage contre les oiseaux ont été assurés selon les besoins afin de minimiser les incidences des ennemis des cultures. Des diguettes antiérosives ont été aménagées tout autour des essais pour limiter les ruissellements.

Tableau 1 : Caractéristiques des différents sites d'essais.

\begin{tabular}{|c|c|c|c|c|c|c|c|c|c|c|}
\hline $\begin{array}{l}\text { Environ } \\
\text { nement }\end{array}$ & Zone & Code & Position & \begin{tabular}{|l} 
Altitude \\
(m)
\end{tabular} & Type sol* & $\begin{array}{l}\text { Pluie } \\
(\mathrm{mm})\end{array}$ & \begin{tabular}{|l} 
Tmin \\
$\left({ }^{\circ} \mathrm{C}\right)$
\end{tabular} & $\begin{array}{l}\text { Tmax } \\
\left({ }^{\circ} \mathrm{C}\right)\end{array}$ & $\begin{array}{l}\text { Date } \\
\text { semis }\end{array}$ & $\begin{array}{l}\text { Précédan } \\
\mathrm{t} \text { cultural }\end{array}$ \\
\hline $\begin{array}{l}\text { Semis } 1 \\
\text { en } 2013\end{array}$ & Bambey & B13D1 & $\begin{array}{l}14^{\circ} 42^{\prime} \mathrm{N} / \\
16^{\circ} 29^{\prime} \mathrm{W}\end{array}$ & 20 & $\begin{array}{l}\text { Sableux }(A=2,3 \%, \\
L=3,5 \%, S=94,2 \%)\end{array}$ & 644 & 23 & 33,9 & $\begin{array}{l}17 / 07 / \\
2013\end{array}$ & Jachère \\
\hline $\begin{array}{l}\text { Semis } 2 \\
\text { en } 2013\end{array}$ & Bambey & B13D2 & & & $\begin{array}{l}\text { Sableux }(A=2,3 \% \text {, } \\
L=3,5 \%, S=94,2 \%)\end{array}$ & 566 & 22,8 & 33,9 & $\begin{array}{l}31 / 07 \mid \\
2013\end{array}$ & Jachère \\
\hline $\begin{array}{l}\text { Semis } 1 \\
\text { en } 2013\end{array}$ & $\begin{array}{l}\text { Sinthiou } \\
\text { Malem }\end{array}$ & S13D1 & $\begin{array}{l}13^{\circ} 49^{\prime} \\
N / 13^{\circ} 55^{\prime} \\
W\end{array}$ & 23 & $\begin{array}{l}\text { Sablo-limoneux } \\
(A=8,0 \%, L=4,0 \% \text {, } \\
S=88,0 \%)\end{array}$ & 575 & 21,4 & 35,3 & $\begin{array}{l}25 / 07 / \\
2013\end{array}$ & Jachère \\
\hline $\begin{array}{l}\text { Semis } 2 \\
\text { en } 2013\end{array}$ & $\begin{array}{l}\text { Sinthiou } \\
\text { Malem }\end{array}$ & S13D2 & & & $\begin{array}{l}\text { Sablo-limoneux } \\
(A=8,0 \%, L=4,0 \% \text {, } \\
S=88,0 \%)\end{array}$ & 536 & 21,2 & 35,4 & $\begin{array}{l}06 / 08 / \\
2013\end{array}$ & Jachère \\
\hline $\begin{array}{l}\text { Semis } 1 \\
\text { en } 2014\end{array}$ & $\begin{array}{l}\text { Sinthiou } \\
\text { Malem }\end{array}$ & S14D1 & & & $\begin{array}{l}\text { Sableux }(A=5,1 \% \text {, } \\
L=5,1 \%, S=89,8 \%)\end{array}$ & 448 & 22,2 & 35,7 & $\begin{array}{l}17 / 07 / \\
2014\end{array}$ & Arachide \\
\hline $\begin{array}{l}\text { Semis } 2 \\
\text { en } 2014\end{array}$ & $\begin{array}{l}\text { Sinthiou } \\
\text { Malem }\end{array}$ & S14D2 & & & $\begin{array}{l}\text { Sableux }(A=5,5 \% \\
L=4,2 \%, S=90,3 \%)\end{array}$ & 377 & 22,1 & 35,6 & $\begin{array}{l}06 / 08 / \\
2014\end{array}$ & Arachide \\
\hline
\end{tabular}

$\mathrm{A}=$ Argile, L= Limon, S=Sable, *Classification selon la méthode USDA faite sur les données moyennes sur l'horizon $0-30 \mathrm{~cm}$ 

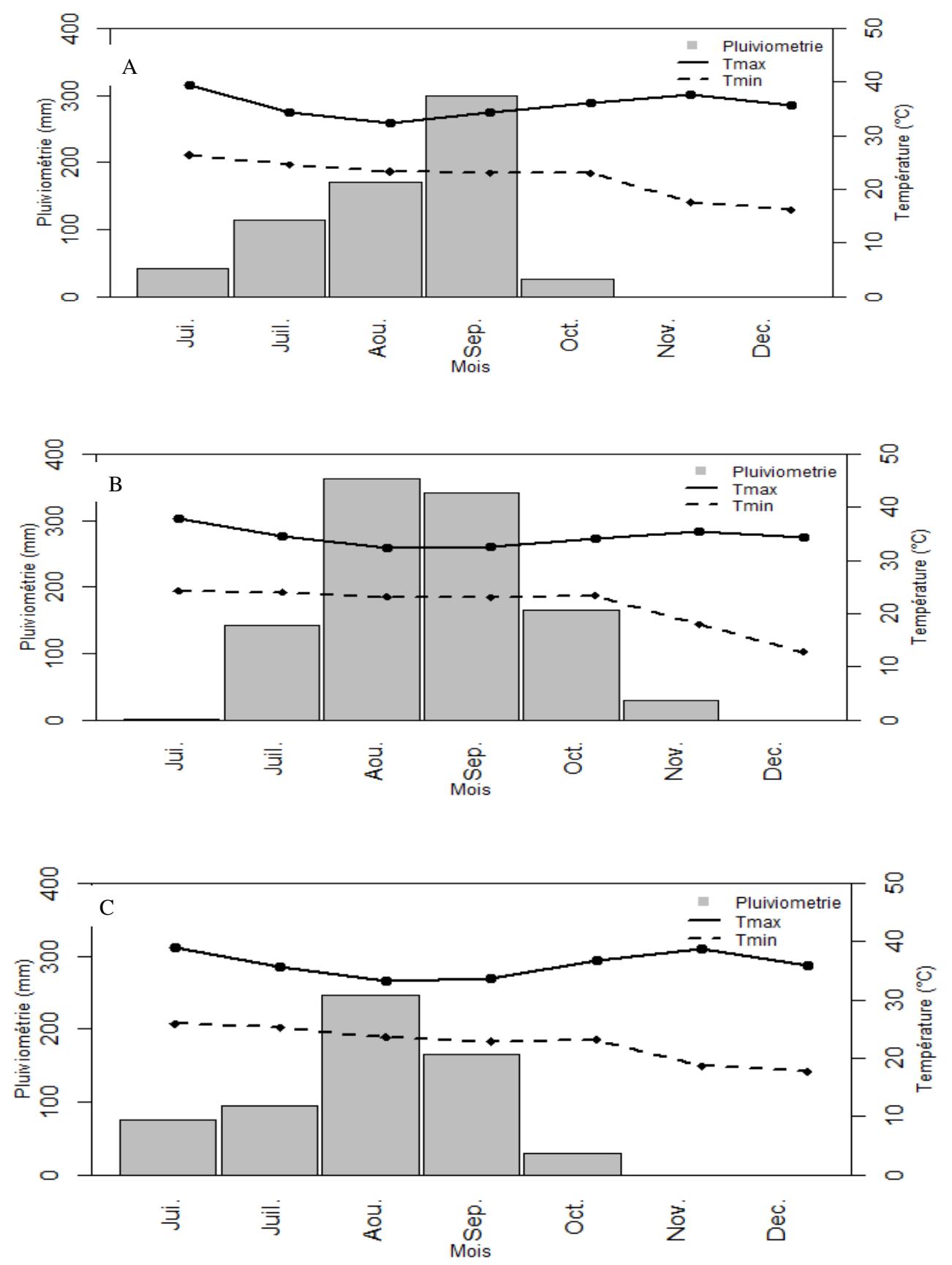

Figure 1: Pluviométrie et températures minimale et maximale des zones d'étude Bambey 2013 (A) et Sinthiou Malem 2013 (B), 2014 (C). Jui.=Juin, Juil= Juillet, Aou= Août, Sep.=Septembre, Oct. =Octobre, Nov. $=$ Novembre, Dec. $=$ Décembre 
Ndiaye et al, J. Appl. Biosci. 2018 Performances agronomiques et stabilité phénotypique de génotypes de Sorgho (Sorghum bicolor (L.) Moench) au Sénégal : une étude des interactions génotypes-environnement

Table 2 : caractéristiques des dix génotypes étudiés.

\begin{tabular}{|c|c|c|c|c|c|c|c|c|c|c|c|}
\hline Génotype & Code & Type & $\begin{array}{l}\text { Sensibilité } \\
\text { photopériode }\end{array}$ & $\begin{array}{l}\text { Longueur } \\
\text { cycle }\end{array}$ & Isohyètes & Finalité & $\begin{array}{l}\text { Hauteu } \\
\text { r plant }\end{array}$ & $\begin{array}{l}\text { Rendement } \\
\text { potentiel }\end{array}$ & $\begin{array}{l}\text { Forme } \\
\text { panicule }\end{array}$ & Autres & Origine \\
\hline Fadda & $\mathrm{G} 1$ & $\begin{array}{l}\text { Guinea } \\
\text { (Hybride) }\end{array}$ & Moyenne & 128 jours & $\begin{array}{l}700-1000 \\
\mathrm{~mm}\end{array}$ & $\begin{array}{l}\text { Grain- } \\
\text { biomasse }\end{array}$ & $2-3 m$ & $4,5 \mathrm{t} / \mathrm{ha}$ & semi-lâche & $\begin{array}{l}\text { tolérant: } \\
\text { moisissure, } \\
\text { anthracnose }\end{array}$ & $\begin{array}{l}\text { Mali, Sélection } \\
\text { IER/ICRISAT, pedigree } \\
\text { 02-SB-F5DT-12A X } \\
\text { Lata. }\end{array}$ \\
\hline Nieleni & G2 & $\begin{array}{l}\text { Guinea } \\
\text { (Hybride) }\end{array}$ & Faible & 115jours & $\begin{array}{l}700-800 \\
\mathrm{~mm}\end{array}$ & Grain & $3 m$ & $4 \mathrm{t} / \mathrm{ha}$ & $\begin{array}{l}\text { Semi- } \\
\text { compacte }\end{array}$ & $\begin{array}{l}\text { tolérant: } \\
\text { moisissure, } \\
\text { anthracnose }\end{array}$ & $\begin{array}{l}\text { Mali, Sélection } \\
\text { IER/ICRISAT }\end{array}$ \\
\hline IS15401 & G3 & $\begin{array}{l}\text { Guinea- } \\
\text { Caudatum }\end{array}$ & Forte & 115 jours & $\begin{array}{l}900-1200 \\
\mathrm{~mm}\end{array}$ & Biomasse & $\begin{array}{l}4-4,5 \\
m\end{array}$ & $2 \mathrm{t} / \mathrm{ha}$ & $\begin{array}{l}\text { semi- } \\
\text { compacte }\end{array}$ & $\begin{array}{l}\text { Résistant : } \\
\text { moisissure, } \\
\text { striga et } \\
\text { cécidomyie }\end{array}$ & $\begin{array}{l}\text { Cameroun, sélection } \\
\text { IER/ICRISAT }\end{array}$ \\
\hline Pablo & G4 & $\begin{array}{l}\text { Guinea } \\
\text { (Hybride) }\end{array}$ & Moyenne & 125 jours & $\begin{array}{l}700-1000 \\
\mathrm{~mm}\end{array}$ & Biomasse & $4 \mathrm{~m}$ & $4 \mathrm{t} / \mathrm{ha}$ & Lâche & $\begin{array}{l}\text { tolérant: } \\
\text { moisissure et } \\
\text { anthracnose }\end{array}$ & $\begin{array}{l}\text { Mali, Sélection } \\
\text { IER/ICRISAT, pedigree } \\
\text { FambeA X Lata. }\end{array}$ \\
\hline CSM63E & G5 & Guinea & Faible & 90 jours & $\begin{array}{l}600-1000 \\
\mathrm{~mm}\end{array}$ & Grain & $4 \mathrm{~m}$ & $2 \mathrm{t} / \mathrm{ha}$ & Lâche & $\begin{array}{l}\text { tolérant: } \\
\text { maladies et } \\
\text { insectes }\end{array}$ & $\begin{array}{l}\text { Mali, variété } \\
\text { traditionnelle }\end{array}$ \\
\hline SK5912 & G6 & Caudatum & Forte & 170 jours & $\begin{array}{l}700-900 \\
\mathrm{~mm}\end{array}$ & biomasse & $2 m$ & $2,5-3,5$ tha & $\begin{array}{l}\text { semi- } \\
\text { compacte }\end{array}$ & $\begin{array}{l}\text { tolérant: } \\
\text { moisissure, } \\
\text { anthracnose }\end{array}$ & Nigéria \\
\hline Grinkan & G7 & Caudatum & Non & 90 jours & $\begin{array}{l}500-800 \\
\mathrm{~mm}\end{array}$ & $\begin{array}{l}\text { Grain- } \\
\text { biomasse }\end{array}$ & $1,2 \mathrm{~m}$ & $4 t / h a$ & $\begin{array}{l}\text { semi- } \\
\text { compacte }\end{array}$ & $\begin{array}{l}\text { résistant: } \\
\text { cécidomyie, } \\
\text { insectes }\end{array}$ & $\begin{array}{l}\text { Mali, sélection } \\
\text { ICRISAT }\end{array}$ \\
\hline Soumba & G8 & Caudatum & Faible & 115 jours & $\begin{array}{l}600-1000 \\
\mathrm{~mm}\end{array}$ & $\begin{array}{l}\text { Grain- } \\
\text { biomasse }\end{array}$ & $2,5 \mathrm{~m}$ & $2,5 \mathrm{t} / \mathrm{ha}$ & $\begin{array}{l}\text { semis- } \\
\text { compacte }\end{array}$ & $\begin{array}{l}\text { tolérant: } \\
\text { maladies et, } \\
\text { insectes, } \\
\text { striga }\end{array}$ & Mali \\
\hline $621 \mathrm{~B}$ & G9 & Caudatum & Non & 105 jours & $\begin{array}{l}600-900 \\
\mathrm{~mm}\end{array}$ & Grain & $1,75 \mathrm{~m}$ & 2,5-3t/ha & $\begin{array}{l}\text { semi- } \\
\text { compacte }\end{array}$ & $\begin{array}{l}\text { résistant aux } \\
\text { moisissures }\end{array}$ & $\begin{array}{l}\text { Sénégal, sélection } \\
\text { ISRA, pedigree CE } \\
151-262 \text { X Sarvato-1 }\end{array}$ \\
\hline $\mathrm{F} 2-20$ & $\mathrm{G} 10$ & Caudatum & Faible & 110 jours & $\begin{array}{l}600-900 \\
\mathrm{~mm}\end{array}$ & Grain & $2,1 \mathrm{~m}$ & 3- $5,3 \mathrm{t} / \mathrm{ha}$ & $\begin{array}{l}\text { semi- } \\
\text { compacte }\end{array}$ & $\begin{array}{l}\text { résistant: } \\
\text { moisissure, } \\
\text { striga }\end{array}$ & $\begin{array}{l}\text { Sénégal, sélection } \\
\text { ISRA, pedigree } \\
\text { (MN1056 x 68-20) X } \\
7410-195-1\end{array}$ \\
\hline
\end{tabular}



Sorgho (Sorghum bicolor (L.) Moench) au Sénégal : une étude des interactions génotypes-environnement

Dispositif expérimental et variables mesurées : Tous les essais ont été conduits selon un même dispositif en blocs complets randomisés avec quatre répétitions (les positions aléatoires des parcelles étant différentes d'un essai à l'autre). Les parcelles élémentaires comprenaient 7 lignes de 40 poquets, pour une superficie de $44,8 \mathrm{~m}^{2}$ $(5,6 \mathrm{~m} \times 8 \mathrm{~m})$, avec en leur centre une placette de 21 poquets ( 3 lignes $\times 7$ poquets; $3,36 \mathrm{~m}^{2}$ ) destinée à l'évaluation des rendements. Les biomasses en paille (feuilles et tiges) et les panicules ont été récoltées à la maturité des grains sur ces placettes. Après battage tous les grains ont été récupérés. Après séchage (à l'air en serre puis $48 \mathrm{~h}$ à l'étuve à $65^{\circ} \mathrm{C}$ ) les poids secs de paille et de grains ont été déterminés pour pouvoir calculer les rendements en grains et en biomasses sèches en kg.ha-1. Analyses statistiques des données : Les données de rendements grains et biomasses paille ont d'abord fait l'objet d'une analyse de la variance par environnement défini dans cette présente étude comme étant une situation expérimentale c'est-à-dire une combinaison " site-année-date de semis » ( 6 en tout) - pour vérifier l'existence d'une différence entre les génotypes. Ensuite une analyse combinée de la variance a été effectuée, considérant l'effet du génotype comme fixe et celui de chaque environnement comme aléatoire selon le modèle statistique suivant :

$$
Y_{i j k}=\mu+G_{i}+E_{j}+B_{k}\left(E_{j}\right)+(G E)_{i j}+\varepsilon_{i j k}
$$

Où $Y_{\mathrm{jik}}$ est le rendement du génotype $\mathrm{i}$ dans l'environnement j et le bloc $k$, $\mu$ est la moyenne générale de tous les rendements, $G_{i}$ est l'effet du génotype $i, E_{j}$ est l'effet de l'environnement $j, B_{k}\left(E_{j}\right)$ est l'effet du bloc $k$ dans l'environnement $j, G_{i j}$ est l'interaction du génotype $i$ avec l'environnement $\mathrm{j}$ et $\varepsilon_{\mathrm{ijk}}$ est l'erreur associée à la moyenne du génotype i dans l'environnement $j$ et dans le bloc $\mathrm{k}$. L'homogénéité des variances résiduelles à travers les environnements a été testée en utilisant le test de Bartlett (Bartlett, 1937). Enfin, l'adaptabilité et les analyses de stabilité phénotypique ont été effectuées par la méthode AMMI comme décrit dans Zobel et al. (1988) en utilisant le modèle statistique suivant :

\section{RESULTATS}

Effets génotype, environnement et génotype $x$ environnement : $L$ 'analyse de la variance combinée est présentée dans le tableau 3. Les effets du génotype, de l'environnement et de l'interaction génotypeenvironnement ont été significatifs pour les rendements en grains et en biomasses $(p<0,001)$. Le rendement moyen en grains des génotypes (tableau 4) a varié de
$Y_{i j}=\mu+g_{i}+\theta_{j}+\sum_{k=n}^{n}\left(\lambda_{k i} \alpha_{i k} y_{j k}+r_{i j}\right)+\varepsilon_{i j}$

Où $Y_{i j}$ est la réponse moyenne du génotype i dans l'environnement j; $\mu$ est la moyenne globale; $g_{i}$ est l'effet fixé du génotype i $(i=1,2, \ldots g)$; $e_{j}$ est l'effet aléatoire de l'environnement $\mathrm{j}(\mathrm{j}=1,2, \ldots \mathrm{e})$; $\varepsilon_{\mathrm{ij}}$ est l'erreur expérimentale; l'interaction $\mathrm{G} \times \mathrm{E}$ est représentée par $\sum_{k=n_{2}}^{n} \lambda_{k} \alpha_{i k} y_{j k}+r_{i j}$

où $\sum$ est la somme des $k=1,2 \ldots k$ axes de l'analyse en composantes principales (PCA) intégrant le modèle, $\lambda_{k}$ est la valeur propre de l'axe $k$, $a_{i k}$ est le vecteur propre du génotype i sur l'axe $k$, $y_{j k}$ est le vecteur propre de l'environnement $\mathrm{j}$ sur l'axe $\mathrm{k}$, et $r_{i j}$ est la résiduelle de l'interaction. La somme des carrés des écarts pour l'interaction $\mathrm{G} \times \mathrm{E}$ a été divisée en $\mathrm{n}$ axes singuliers ou composants principaux d'interaction (IPCA), qui a été décrite par la partie standard, chaque axe correspondant à un modèle AMMI. Généralement, lorsque les interactions $G \times E$ sont significatives, les modèles avec un $\left(A M M_{1}\right)$ ou deux $\left(A M M_{2}\right)$ axes principaux sont les plus utilisés à cause de leur simplicité qu'on peut représenter en graphe biplot (c'est-à-dire sur un type de graphique qui contient deux catégories de points). Dans notre étude, il s'agit de génotypes et d'environnements. L'interprétation du graphique biplot a été basée sur la variation causée par les effets principaux additifs du génotype et de l'environnement et l'effet multiplicatif de l'interaction $G \times$ E. Selon Zobel et al. (1988) pour le graphe $\mathrm{AMM}_{2}$, les génotypes, qui ont de faibles scores sur l'IPCA ${ }_{1}$ (premier axe de l'analyse en composante principale de l'interaction) ou l'IPCA 2 (deuxième axe de l'analyse en composante principale de l'interaction) ou les deux à la fois, contribuent faiblement à l'interaction, et cela indique une adaptation générale. Par contre ceux qui ont des scores élevés, positifs ou négatifs, présentent de fortes interactions, et sont spécifiquement adaptés à l'environnement qui a le score de même signe. Toutes les analyses statistiques ont été effectuées en utilisant le logiciel $R$ version 3.2 (R Développement Core Team, 2015).

$1854 \mathrm{Kg} \mathrm{ha}^{-1}$ (Nieleni) à $547 \mathrm{Kg}$ ha-1 $^{-1}$ (SK5912). Les génotypes ont montré des performances inconstantes à travers les environnements sauf Fadda et Nieleni qui étaient relativement bons dans tous les environnements (tableau 4). Quatre génotypes ont obtenu un rendement moyen supérieur à la moyenne générale (1320 Kg.ha-1) à savoir Nieleni, Fadda, Pablo et 621B avec des 

Sorgho (Sorghum bicolor (L.) Moench) au Sénégal : une étude des interactions génotypes-environnement

rendements respectifs de $1814 \mathrm{Kg}_{\mathrm{ha}} \mathrm{ha}^{-1}, 1743 \mathrm{Kg} \cdot \mathrm{ha}^{-1}$, $1601 \mathrm{Kg} \mathrm{ha}^{-1}$ et $1330 \mathrm{Kg} \cdot \mathrm{ha}^{-1}$. Parmi ces génotypes, Nieleni et Fadda ont montré des rendements élevés pour les différents environnements (tableau 4). Les trois génotypes les moins performants ont été Soumba, Grinkan et SK5912 avec des rendements grains moyens respectifs de $1168 \mathrm{Kg}^{-h^{-1}}, 1051 \mathrm{Kg} \cdot \mathrm{ha}^{-1}$ et $547 \mathrm{Kg} \cdot \mathrm{ha}^{-1}$. En outre, le rendement moyen en grain des environnements (tableau 4) a varié de $530 \mathrm{Kg}^{-h^{-1}}$ (B13D2) à $1714 \mathrm{Kg}^{-h a^{-1}}$ (S13D1). Trois environnements sur les six ont eu des rendements moyens supérieurs à la moyenne générale: S13D1 (1714 Kg.ha-1), S14D1 (1696 Kg.ha-1), S13D2 (1570 Kg.ha-1). En ce qui concerne la biomasse paille (tableau 5), les rendements des génotypes ont varié de $12103 \mathrm{Kg}_{\text {gha-1 }}{ }^{-1}$ (IS15401) à $4647 \mathrm{Kg}$ ha $^{-1}$ (CSM63E). Les génotypes ont montré des performances inconstantes dans tous les environnements sauf IS15401 et SK5912 qui étaient relativement bons dans tous les environnements (tableau 5). Quatre des six génotypes ont obtenu des rendements moyens audessus de la moyenne générale (7603 Kg.ha-1) à savoir IS15401, SK5912, Fadda et Nieleni, avec des valeurs respectives de $12103 \mathrm{Kg} \cdot \mathrm{ha}^{-1}, 11156 \mathrm{Kg}^{-h^{-1}}, 8018 \mathrm{Kg} \cdot \mathrm{ha}-$ ${ }^{1}$ et de 7935 Kg.ha-1 $^{-1}$. Parmi eux SK5912 et IS15401 ont obtenu des rendements élevés pour plusieurs environnements. Le rendement moyen en biomasse paille des environnements (tableau 5) a varié entre $5742 \mathrm{Kg}$.ha${ }^{1}$ (S13D2) et $9536 \mathrm{Kg} \mathrm{ha}^{-1}$ (B13D2). Trois environnements sur les six ont des rendements en dessus de la moyenne générale, en l'occurrence B13D2, S14D1 et B13D1 avec des rendements respectifs de $9536 \mathrm{Kg} \cdot \mathrm{ha}^{-1}, 9015 \mathrm{Kg} \mathrm{ha}^{-1}$, $8055 \mathrm{Kg} \mathrm{ha}^{-1}$.

Tableau 3 : Résumé de l'analyse de variance combinée et de la décomposition de l'interaction $\mathrm{G} \times \mathrm{E}$ selon $\mathrm{AMMI}$.

\begin{tabular}{l|c|c|c|c|c}
\hline Source de variation & DL & \multicolumn{2}{|c|}{ Grains (Kg.ha-1) } & \multicolumn{2}{c}{ Biomasses paille (Kg.ha-1) } \\
\cline { 3 - 6 } & & Carrée Moyen & $\begin{array}{c}\text { Variance expliquée } \\
(\%)\end{array}$ & Carrée Moyen & $\begin{array}{c}\text { Variance expliquée } \\
(\%)\end{array}$ \\
\hline Génotype (G) & 9 & $3409943^{* * *}$ & 32,8 & $144371591^{* * *}$ & 47,7 \\
Environnement (E) & 5 & $7712115^{* * *}$ & 41,2 & $89472372^{* *}$ & 16,4 \\
Blocs (E) & 18 & $352673^{*}$ & 6,8 & $19792457^{\star * *}$ & 13,1 \\
Interaction (G x E) & 45 & $400170^{* * *}$ & 19,2 & $13814779^{* * *}$ & 22,8 \\
IPCA $_{1}$ & 13 & $733183^{* *}$ & 54,3 & $23600674^{* * *}$ & 59,3 \\
IPCA $_{2}$ & 11 & $389402^{*}$ & 24,4 & $11512314^{*}$ & 24,5 \\
IPCA $_{3}$ & 9 & 249314 & 12,8 & 5892251 & 10,3 \\
IPCA $_{4}$ & 7 & 121655 & 4,9 & 3881248 & 5,3 \\
PCCA $_{5}$ & 5 & 129493 & 3,7 & 708295 & 0,7 \\
Erreur & 157 & 188075 & & 5497548 & \\
\hline DL & & & & \\
\hline
\end{tabular}

$\mathrm{DL}=$ degré de liberté $;{ }^{* * *},{ }^{* *},{ }^{*}=$ significatif au seuil de $0,1 \%$ et $1 \%$ et $5 \%$

Tableau 4 : Rendement moyen en grains $\left(\mathrm{Kg}_{\mathrm{g}} \mathrm{ha}^{-1}\right)$ des dix génotypes sur les dix environnements étudiés.

\begin{tabular}{|c|c|c|c|c|c|c|c|}
\hline \multirow[t]{2}{*}{ Génotype } & \multicolumn{7}{|c|}{ Environnements } \\
\hline & B13D1 & B13D2 & S13D1 & S13D2 & S14D1 & S14D2 & Moyenne \\
\hline FADDA & $1662^{\mathrm{ab}}$ & $804^{\mathrm{ab}}$ & $2329^{a b}$ & $1855^{a}$ & $1604^{\mathrm{ab}}$ & $2206^{a}$ & 1743 \\
\hline NIELENI & $2011^{a}$ & $972^{\mathrm{a}}$ & $2445^{\mathrm{a}}$ & $\overline{1742^{\mathrm{ab}}}$ & $2626^{a}$ & $1326^{\mathrm{abc}}$ & $\overline{1854}$ \\
\hline IS15401 & $665 b^{c}$ & $554^{\mathrm{bc}}$ & $2182^{a b}$ & $1608^{a b}$ & $1297^{\mathrm{b}}$ & $1008^{b c}$ & $\overline{1219}$ \\
\hline PABLO & $1688^{\mathrm{ab}}$ & $624^{b c}$ & $2111^{a b}$ & $1786^{\mathrm{ab}}$ & $1592^{\mathrm{ab}}$ & $1806^{\mathrm{ab}}$ & 1601 \\
\hline CSM63E & $\overline{1423^{a b c}}$ & $346^{\mathrm{cd}}$ & $1628^{a b c}$ & $1895^{\mathrm{a}}$ & $1918^{a b}$ & $\overline{1634^{\mathrm{ab}}}$ & 1474 \\
\hline SK5912 & $252^{c}$ & $151^{d}$ & $992^{c}$ & $\frac{1071^{b}}{4}$ & $459 c$ & $358^{c}$ & 547 \\
\hline GRINKAN & $888^{a b c}$ & $502^{\mathrm{bc}}$ & $1323^{\mathrm{bc}}$ & $1475^{\mathrm{ab}}$ & $1677^{a b}$ & $441^{c}$ & 1051 \\
\hline SOUMBA & $981 \mathrm{abc}$ & $553^{\mathrm{bc}}$ & $1016^{c}$ & $1301^{a b}$ & $2307^{a b}$ & $900^{\text {bc }}$ & 1168 \\
\hline $621 B$ & $1367^{a b c}$ & $491^{b c}$ & $1566^{a b c}$ & $1665^{\mathrm{ab}}$ & $\overline{1827^{a b}}$ & $1064^{b c}$ & 1330 \\
\hline$F 2-20$ & $1223^{a b c}$ & $409 \mathrm{~cd}$ & 1549 abc & $1302^{\mathrm{ab}}$ & $1658^{\mathrm{ab}}$ & $1110^{b c}$ & 1208 \\
\hline Moyenne & 1211 & 530 & 1714 & 1570 & 1696 & 1192 & 1320 \\
\hline
\end{tabular}

Pour chaque colonne, les moyennes de même lettre ne sont pas significativement différentes au seuil de $5 \%$. Les valeurs en gras indiquent les deux meilleurs génotypes (environnements) en termes de rendement grain. 
Ndiaye et al, J. Appl. Biosci. 2018 Performances agronomiques et stabilité phénotypique de génotypes de Sorgho (Sorghum bicolor (L.) Moench) au Sénégal : une étude des interactions génotypes-environnement

Tableau 5 : Rendement moyen en biomasses paille $\left(\mathrm{Kg}_{\mathrm{H}} \mathrm{ha}^{-1}\right)$ des dix génotypes dans les dix environnements étudiés.

\begin{tabular}{|c|c|c|c|c|c|c|c|}
\hline \multirow{2}{*}{ Génotype } & \multicolumn{7}{|c|}{ Environnements } \\
\hline & B13D1 & B13D2 & S13D1 & S13D2 & S14D1 & S14D2 & Moyenne \\
\hline FADDA & 11111 abc & $10546^{\mathrm{abc}}$ & $7571^{b}$ & $5409^{a b c}$ & $8900^{c}$ & $4568^{b c}$ & 8018 \\
\hline NIELENI & $7667^{a b c}$ & $13322^{\mathrm{ab}}$ & $5617^{\mathrm{bcd}}$ & $6473^{a b c}$ & $7552^{c}$ & $6981 \mathrm{abc}$ & 7935 \\
\hline IS15401 & $12315^{\mathrm{ab}}$ & $15989 a$ & $10611^{a}$ & $7151^{\mathrm{ab}}$ & $17679 \mathrm{a}$ & $8871^{a}$ & $\underline{12103}$ \\
\hline PABLO & $8137 \mathrm{abc}$ & $7198 b^{c d}$ & $7109 \mathrm{bc}$ & $5775^{\mathrm{abc}}$ & $8097^{c}$ & $6263 \mathrm{abc}$ & 7096 \\
\hline CSM63E & $3642^{c}$ & $3051^{d}$ & $4926^{\mathrm{bcd}}$ & $4529 c$ & $7712^{c}$ & $4020^{c}$ & 4647 \\
\hline SK5912 & $14806^{a}$ & $13623^{a b}$ & 9827a & $7480^{a}$ & $12417^{b}$ & $8783^{a}$ & 11156 \\
\hline GRINKAN & $\overline{9675^{\mathrm{abc}}}$ & $12020^{\mathrm{abc}}$ & $6870^{\mathrm{bcd}}$ & $4812^{\mathrm{bc}}$ & $8428^{c}$ & $5010^{a b c}$ & $\overline{7803}$ \\
\hline SOUMBA & $4756^{b c}$ & $7497^{\mathrm{bcd}}$ & $4034^{d}$ & $5109^{b c}$ & $7270^{c}$ & $5884^{a b c}$ & 5758 \\
\hline $621 \mathrm{~B}$ & $3581^{c}$ & $5955^{c d}$ & $4300^{\text {cd }}$ & $4889^{b c}$ & $4239 c$ & $6982^{a b c}$ & 4991 \\
\hline F2-20 & $4863^{b c}$ & $721 \mathrm{gbcd}^{\mathrm{b}}$ & $5261^{\mathrm{bcd}}$ & $5791^{a b c}$ & $7855^{c}$ & $8171^{a b}$ & 6527 \\
\hline Moyenne & 8055 & 9536 & 6613 & 5742 & 9015 & 6531 & 7603 \\
\hline
\end{tabular}

Pour chaque colonne, les moyennes de même lettre ne sont pas significativement différentes au seuil de $5 \%$. Les valeurs en gras indiquent les deux meilleurs génotypes (environnements) en termes de rendement biomasse paille.

Analyse AMMI de l'interaction génotypeenvironnement ( $G \times E)$ : L'analyse AMMI de la variance à travers les six environnements a montré pour le rendement grains que $32,8 \%, 41,2 \%$ et $19,2 \%$ de la somme totale des carrés des écarts ont été attribués respectivement aux génotypes, aux environnements et à l'interaction génotype-environnement. Pour le rendement en biomasses, une grande partie de la variabilité observée $(54,0 \%)$ a été attribuée aux génotypes et seulement $16,4 \%$ et $22,8 \%$ aux environnements et à l'interaction génotype-environnement. Pour la décomposition de l'interaction $G \times E$ selon le modèle AMMI, l'analyse a montré que les deux premières composantes principales de l'interaction ont été significatives (tableau 3) pour les deux rendements, et qu'elles expliquent respectivement $78,7 \%$ et $83,8 \%$ de la somme des carrés des écarts pour le rendement grain et le rendement biomasse paille (IPCA 1 et IPCA $)$. Ces résultats ont indiqué que les scores des génotypes et des environnements sur les deux premières composantes principales de l'interaction expliquent la presque totalité de l'interaction qui s'est présentée dans la matrice des données.

Réponse et stabilité des génotypes selon le modèle AMMI : Le graphe biplot $\mathrm{AMMI}_{2}$ montre pour le rendement grain que les environnements S14D1, S14D2 et S13D1, du fait de leurs scores élevés, ont le mieux discriminé les performances des différents génotypes évalués et ontété une source significative de contribution à l'interaction (figure 2A). Cependant leurs moyennes à l'exception de S14D2 ont été parmi les plus élevés, ce qui indique que ce sont des environnements favorables pour obtenir des moyennes élevées. Par contre, B13D1, S13D2 et B13D2 ont contribué nettement moins à l'interaction et ont obtenu les plus faibles rendements à l'exception S13D2. De par leur position le long des deux axes (scores proches de zéro), $621 \mathrm{~B}$ et F2-20 ont été les plus stables, et ils présentent un rendement inférieur à la moyenne générale pour $\mathrm{F} 2-20$ et très proche de la moyenne pour 621B. Par contre Fadda et Pablo, tous avec des rendements supérieurs à la moyenne générale, se sont montrés très instables du fait de leurs positions très éloignées de l'origine, avec une adaptation spécifique à l'environnement S14D2. II en est de même pour SK5912 et IS15401 vis-à-vis de l'environnement S13D1 et Soumba pour S14D1, avec tous avec des rendements inférieurs à la moyenne générale. En ce qui concerne le rendement en biomasse, les environnements B13D1, B13D2, S13D2, S14D1 et S14D2 ont contribué fortement à l'interaction suite à leurs positions très éloignées de l'origine (figure 2B). Parmi ces environnements fortement interactifs, B13D1, B13D2 et S14D1 ont enregistré des rendements au-dessus de la moyenne globale. S13D1 s'est distingué par une faible contribution à l'interaction comme l'indique sa position proche de l'origine avec un rendement moyen au-dessus de la moyenne globale. Deux génotypes Nieleni et Pablo se sont révélés plus stables vu leurs positions proches de l'origine et parmi eux seul Nieleni a eu des rendements supérieurs à la moyenne générale. A l'opposé SK5912 et Fadda se sont révélés très instables, c'est-à-dire donc très sensibles aux interactions environnementales, avec des adaptations spécifiques à l'environnement B13D1 (figure 2B). Il en est de même pour IS15401 vis-à-vis de l'environnement S14D1. Par ailleurs ces trois génotypes ont obtenus des rendements supérieurs à la moyenne générale. 



Figure 2 : Biplot $\mathrm{AMMl}_{2}$ des dix génotypes de sorgho et les six environnements (combinaison site-date de semisannée au Sénégal) pour les rendements grains (A) et biomasses paille (B).B13D1: date semis 1 Bambey, B13D2: date semis 2 Bambey, S13D1 : date semis 1 Sinthiou Malem, S13D2 : date semis 2 Sinthiou Malem, S14D1 : date semis 1 Sinthiou Malem, S14D2 : date semis 2 Sinthiou Malem. 

Sorgho (Sorghum bicolor (L.) Moench) au Sénégal : une étude des interactions génotypes-environnement

\section{DISCUSSION}

L'analyse de la variance combinée a révélé des effets significatifs pour l'environnement, les génotypes et l'interaction génotype-environnement. Ceci confirme une diversité phénotypique parmi les génotypes évalués et que ces génotypes répondent différemment aux environnements. Ces résultats sont du même type que ceux d'Almeida Filho et al. (2014) et Showemimo et al. (2000) sur le sorgho. Les rendements des dix génotypes ont varié entre $547 \mathrm{Kg}$ ha $^{-1}$ et $1854 \mathrm{Kg} \cdot \mathrm{ha}^{-1}$ pour le grain et entre $4647 \mathrm{Kg}$ ha- $^{-1}$ et $12103 \mathrm{Kg}$.ha-1 pour la biomasse ; et ceux des environnements, entre $541 \mathrm{Kg}^{-h^{-1}}$ et $1696 \mathrm{Kg} \mathrm{ha}^{-1}$ en grain et entre $5742 \mathrm{Kg}^{-h a^{-1}}$ et $9642 \mathrm{Kg}$ ha- $^{-1}$ en biomasse (tableaux 4 et 5). D'une manière générale les rendements sont plus importants en date 1 de semis qu'en date 2, montrant l'importance potentielle d'une durée de cycle plus longue et l'existence d'une réponse à la photopériode des sorghos étudiés, comme cela a déjà pu être mis en évidence en Afrique de l'Ouest (Kouressy et al., 2008). Les faibles rendements grain observés à Bambey en 2013 (B13D1 et B13D2) età Sinthiou Malem en 2014 (S14D2) pourraient être expliqués entre autres par le caractère particulièrement sableux du sol, et par un complexe de maladies et/ou carences (signes observés mais non clairement identifiés), contraintes auxquelles les génotypes 621B, Grinkan, Soumba et F2-20 se sont montrés bien plus sensibles. A noter que ce sont tous des Caudatum qui sont des génotypes améliorés introduits au niveau des programmes nationaux de sélection du sorgho et qui sont connus pour être moins rustiques que les Guinea lorsque les conditions édaphiques ne sont pas idéales (Bazile et al., 2008). Pour les essais de Bambey des stress hydriques en fin de cycle (découlant de problèmes avec le système d'irrigation) sont également survenus. Ils expliquent le très faible rendement du SK5912, et ils ont pu aggraver les effets des contraintes précédemment mentionnées. À l'heure actuelle, le modèle le plus utilisé est l'AMMI (Raton et al., 1992; Raju, 2002; Zali et al., 2012), qui implique à la fois l'Anova et l'analyse en composante principale afin de décomposer l'interaction $G$ $x E$. Dans cette présente étude, les deux premières composantes principales de l'interaction ont été significatives (tableau 3) pour les deux rendements, et ont expliqué respectivement $78,7 \%$ et $83,8 \%$ de la somme des carrés des écarts pour le rendement grain et le rendement biomasse paille (IPCA 1 et IPCA $)$. Pour le grain, la première composante $\left(\mathrm{IPCA}_{1}\right)$ a été fortement corrélée à la bonne fertilité du sol (S14D1 et S13D1) alors que la deuxième composante l'a été pour la pluviométrie (faible en S14D2). En ce qui concerne la biomasse paille,
I'IPCA 1 a été fortement corrélé positivement à B13D1 et B13D2 (bonne production malgré la maladie et le stress en fin cycle en B13D2 qui n'a pas affecté la biomasse) et négativement à S14D2 et S13D2 (bonne fertilité et dates tardives) alors que l'IPCA 2 a été positivement corrélé à S14D1 (bonne fertilité et faible pluviométrie). Deux génotypes pour le grain (621B, F2-20) et deux génotypes pour la biomasse (Nieleni et Pablo) ont été identifiés comme globalement plus stables selon ce modèle, mais ils ont par ailleurs montré, à l'exception de Nieleni, des rendements inférieurs à la moyenne à travers les différents environnements. Ces résultats corroborent ceux de Ménad et al. (2010) qui stipulent que la stabilité des rendements est indépendante de leurs valeurs, et que les génotypes au rendement élevé sont en général relativement peu stables alors que c'est généralement le contraire pour les génotypes au rendement moyen ou faible. Ils confirment aussi l'affirmation de Yan et Hunt (2002) qui indiquent que la stabilité globale n'est pas forcément un facteur positif et qu'elle n'est souhaitable que lorsqu'elle associe un rendement moyen élevé. En plus, l'analyse AMMI a révélé aussi pour le grain que les génotypes IS15401 et SK5912, Fadda et Pablo, Soumba ont été proches respectivement des environnements S13D1, S14D2 et S14D1 caractérisés par une bonne fertilité des sols alors que pour la biomasse les génotypes IS15401, SK5912 et Fadda ont été proches respectivement des environnements S14D1 et B13D1 caractérisé par une date de semis précoce, ce qui indique des adaptations spécifiques. A l'opposé, IS15401 était très peu adaptés aux environnements S14D2, S13D2, et il en a été de même pour SK5912 vis-à-vis de S14D2 pour la biomasse. Les adaptations spécifiques des génotypes de cycle long IS15401 et SK5912 à l'environnement S13D1 et de Soumba à S14D1 pourraient être dues à la fois aux dates de semis normale et aux bonnes conditions agro-pédologiques qui ont permis à ces génotypes d'enregistrer logiquement de meilleurs performances même si S14D1 a été moins arrosé (tableau 1). Les très faibles adaptations de IS15401 et SK5912 aux environnements S13D2 et S14D2 pour la biomasse pourraient être imputées d'une part aux dates de semis tardives et d'autre part à la faible pluviométrie enregistrée dans ces environnements. La possibilité d'identifier des génotypes stables et des génotypes avec des adaptations spécifiques à certains environnements constituent un avantage important de la méthode AMMI comparativement aux autres méthodes couramment employées (Silva Filho et al., 2008). 


\section{CONCLUSION}

Le rendement des cultures est un trait complexe qui est influencé par un certain nombre de facteurs associés à l'environnement, directement ou indirectement rendant difficile la sélection de sorghos stables à haut rendement pour divers environnements. Dans la présente étude, le modèle AMMI a montré que les rendements grains et biomasses ont fortement été influencés par les génotypes, les environnements et l'interaction génotypeenvironnement. Les environnements ont provoqués des réponses différentes des génotypes et la plupart de ces génotypes ont montré une spécificité environnementale. Nieleni et Fadda ont eu les rendements en grains les plus élevés en moyenne alors que IS15401 et SK5912 ont eu

\section{REMERCIEMENTS}

Le premier auteur remercie le Centre d'Étude Régional pour l'Amélioration à l'Adaptation à la Sécheresse (CERAAS) qui lui a accordé cette opportunité pour la préparation de sa thèse de doctorat. Les travaux de terrain ont été financés par le PPAAO/WAAPP (Programme de Productivité Agricole en Afrique de l'Ouest) à travers le Projet CERAAS (Développement de variétés de céréales adaptées aux conditions agro-

\section{REFERENCES BIBLIOGRAPHIQUES}

Agrama, H.A. and Tuinstra, M.R., 2003. La diversité phylogénétique et les relations entre le sorgho adhésions à l'aide SSR et RAPD. Africaine. J. Biotechnol., 2 (10) : 334-340.

Almeida Filho, J.E., Tardin, F.D., Daher1, R.F., Barbé, T.C., Paula3, C.M., Cardoso, M.J., Godinho V.P.C., 2014: Stability and adaptability of grain sorghum hybrids in the off-season Genet. Mol. Res., 13 (3): 7626-7635.

Ba, K., Tine, E., Destain, J., Cissé, N., Thonart, P., 2010. Étude comparative des composés phénoliques, du pouvoir antioxydant de différentes variétés de sorgho sénégalais et des enzymes amylolytiques de leur malt. Biotechnol. Agron. Soc. Environ., 14: 131-139.

Bartlett, M. S., 1937. Properties of sufficiency and statistical tests. Proceedings of the Royal Statistical Society. Series A, 160: 268-282.

Bazile, D., Dembélé, S., Soumaré, M., Dembele, D., 2008. Utilisation de la diversité variétale du sorgho pour valoriser la diversité des sols au Mali. Cahiers Agricultures, $17: 86-94$.

Crossa, J., Gauch, H.G., Zobel, R.W., 1990. Additive main effect and multiplicative interaction analysis les rendements les plus élevés en biomasse. Les génotypes F2-20 pour le grain et Pablo pour la biomasse ont été identifiés comme des génotypes avec une bonne stabilité phénotypique mais de faibles performances. Par contre les génotypes Fadda et Pablo, IS15401 et SK5912 pour le grain ont montré des adaptations spécifiques respectivement aux environnements S14D2 et S13D1. Pour la biomasse paille les cycles longs SK5912 et IS15401 ont été spécifiquement adaptés aux environnements B13D1 et S14D1 tous semés en date normale. Le modèle statistique AMMI peut être un excellent outil pour aider à recommander des génotypes de sorgho comme d'autres cultures.

écologiques en Afrique de l'Ouest et répondant aux besoins des utilisateurs) et suivis au quotidien par Gabriel Diatta et M'Baye Sarr Diop. Nos remerciements vont également à l'endroit des équipes techniques du CERAAS à Bambey et de la station de Recherches ISRA/Sinthiou Malem (Sénégal) et à tous les autres agents desdits services.

of two international maize cultivar trials. Crop Science, 30: 493-500.

Djè, Y., Heuertz, M., Ater, M., Lefebvre, C., Vekemans, X., 2007. Évaluation de la diversité morphologique des variétés traditionnelles de sorgho du Nord-ouest du Maroc. Biotechnol. Agron. Soc. Environ., 11 : 30-40.

FAOSTAT., 2015. Statistiques des données année 2015, Rome, Italy. United Nations Food and Agriculture Organization, Rome.

Gauch, H.G.; Zobel, R.W., 1996. AMMI analysis of yield trials. Chap. 4. p. 85-122. In: Kang, M.S.; Gauch, H.G., eds. Genotype by environment interaction. CRC Press, Boca Raton, FL, USA.

Holhs T., 1995. Analysis of genotype environment interactions. South Afric J Sci., 91: 121-124.

Kempton, R.A., 1984. The use of biplots in interpreting variety by environment interactions. Journal of Agricultural Science, 103: 123-135.

Kouressy, M., Dingkuhn, M., Vaksmann, M. and Heinemann, A.B., 2008. Adaptation to diverse semi-arid environments of sorghum genotypes having different plant type and sensitivity to photoperiod. Agric. For. Meteorol., 148: 357-371. 
Menad, A., Meziani, N., Bouzerzour, H. and Benmahammad, A., 2010. Analyse de l'interaction génotype $x$ milieux du rendement de l'orge (Hordeum vulgare L.) : application des modèles AMMI et la régression conjointe. Nature \& Technologie, 05: 99-106.

Raju, B.M.K., 2002. Study of AMMI model and its biplots. Journal of the Indian Society of Agricultural Statistics 55(3), 297-322.

Raton, F.L., Gauch, H.G., 1992. Statistical analysis of regional yield trials. AMMI Analysis of Factorial Designs. Elsevier, New York.

R Development Core Team., 2015. R : A Language and Environment for Statistical Computing. R Foundation for Statistical Computing. Available at [http://www.R-project.org/] Accessed December 18, 2015, Vienna.

Romagosa, I., Fox, P.N., 1993. Genotype x environment interaction and adaptation. In : Plant Breeding : Principles and Prospects. (Hayward, M.D., Bosemark, N.O., Romagosa, l., eds), Chapman \& Hall, Londres pp : 373-390.

Seguin, B., Soussana, J.F., 2008. Émissions de gaz à effet de serre et changement climatique : causes et conséquences observées pour l'agriculture et l'élevage. Courrier de l'environnement de l'INRA, 55: 79-91.

Showemimo, F.A., Echekwu, C.A., Yeye, M.Y., 2000. Genotype x environment interaction in Sorghum trials and their implication for future variety évaluation in Sorghum growing areas of northern Nigeria. The PIt. Scientist, 1: 24-31.

Silva Filho, J.L., Morello, C.L., Farias, F.J.C., Lamas, F.M., 2008. Comparação de métodos para avaliar a adaptabilidade eestabilidade produtiva em algodoeiro. Pesq. Agropec. Bras., 43: 349355.

Vachaud, G., Dancette, C., Sonko, S., Thony, J.L., 1978. Méthode de caractérisation hydrodynamique d'un sol non saturé. Application à deux types de sols du Sénégal. Annales Agronomiques vol. 29, No1: 1-36.

Yan, W. and Hunt, L.A., 2002. Biplot analysis of multienvironment trial data. In Kang MS (ed.). Quantitative Genetics, Genomics and Plant Breeding. Louisiana State University, Louisiana pp : 289-304.

Zali, H., Farshadfar, E., Sabaghpour, S.H., Karimizadeh, R., 2012. Evaluation of genotype $\times$ environment interaction in chickpea using measures of stability from AMMI model. Annals of Biological Research, 3 (7) : 3126-3136.

Zobel, R.W., Wright, M.W. and Gauch, H.G., 1988. Statistical analysis of a yield trial. Agron J., 80: $388-39$ 